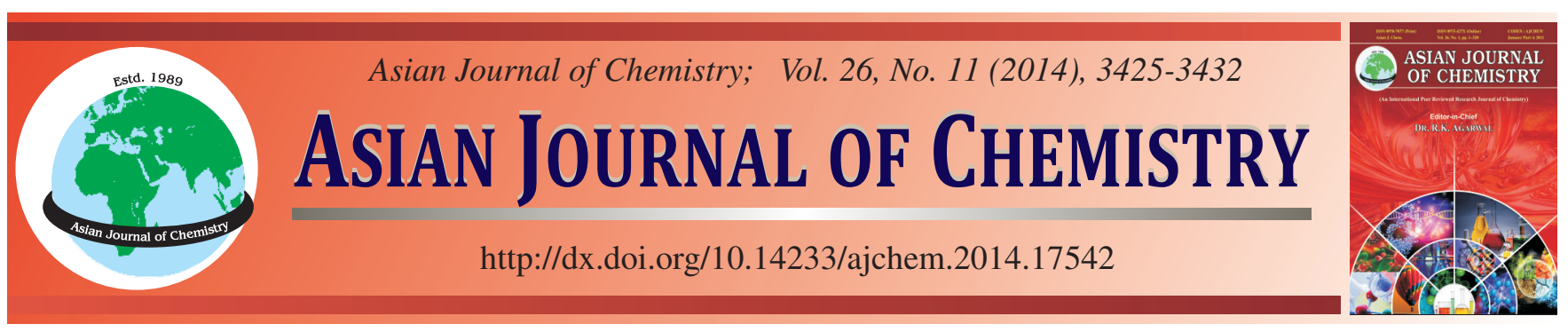

\title{
Neuron-Like Differentiation of Mesenchymal Stem Cells Mediated by Nurr1 and Mash1 in vitro
}

\author{
Xiao-Long Tang ${ }^{1,2}$, Zhen Huo ${ }^{1}$, Li-Fa Xu ${ }^{1,2}$, Xiu-Yun Zhang $^{1}$, Yi-Lin Yin ${ }^{1}$, Lin Sun ${ }^{1}$, \\ Rong-Bo Zhang ${ }^{1}$, Le-Lin Hu ${ }^{1}$, Shu-Yu Cai ${ }^{1,2}$, Zhen-You Jiang ${ }^{3}$, Yi Zheng ${ }^{4}$ and Tian-Yi Chen ${ }^{1, *}$
}

${ }^{1}$ Stem Cell Engineering Research Center, School of Medical, Anhui University of Science \& Technology, Huinan 232001, P.R. China ${ }^{2}$ Department of Laboratory Medicine, Tianjin Medical College, Tianjin 300222, P.R. China

${ }^{3}$ Department of Microbiology and Immunology, School of Medical, Jinan University, Guangzhou 510632, P.R. China

${ }^{4}$ School of Life Sciences, Tsinghua University, Beijing 100084, P.R. China

*Corresponding author: Tel: +86 554 6668770; E-mail: txljd2006@126.com

\begin{abstract}
Cellular therapies using stem cells are promising approaches for the treatment of neurological diseases, as neuron has limited potential to self-renew or repair. Thus, mesenchymal stem cells (MSCs), a kind of multipotent progenitors, can represent an alternative source of cells for neuron replacement therapies. Here we show that forced co-expression of neurogenic factors Nurr1 and Mash1, two transcriptional factors specific to dopamine neuron development and treatment with Neurobasal-A medium containing nerve growth factor and GSK-3 inhibitors (TWS119) could directly and effectively induce mesenchymal stem cells differentiation into morphologically, phenotypically and functionally neuron-like cells. The differentiated cells significantly increased expression of neuron specific transcription factors (Pitx3, Lmx1a, Ngn2 and En2); increased various neuron markers such as tyrosine hydroxylase, microtubule associated protein-2 (MAP2), dopamine transporter (DAT), neuron-specific neuronal protein (NeuN), neurofilament medium (NF-M), $\beta$-III Tubulin (Tuj1) and neuron specific enolase (NSE). Our results showed characteristic dopamine neurotransmitter release in response to depolarization stimuli and specific dopamine reuptake properties in vitro. Nurr1 and Mash1 expression remained the number of dopamine neuron-like cells stable in the cultures for 15 days, implying that Mash1 and Nurr1 modulate neuron-like differentiation of mesenchymal stem cells. Altogether, the combined expression of specific transcription factors Nurr1 and Mash1 enhances mesenchymal stem cells differentiation in vitro to generate neuron-like cells as a promising source of cell for therapy of neurological diseases.
\end{abstract}

Keywords: Dopaminergic neurons, Mesenchymal stem cells, Differentiation, Transcriptional factor.

\section{INTRODUCTION}

Mesenchymal stem cells (MSCs) are attractive for the regeneration of damaged tissues in clinical applications, since they are able to self-renew with a high proliferative capacity and possess multidifferentiation potential that can be demonstrated in vitro ${ }^{1}$. So MSCs hold potential in advancing the treatment of many diseases and disorders that are currently untreatable ${ }^{1-4}$, in particular, neural injuries and degenerated diseases are characterized by a loss of neurons, as neural tissue is understood to have a limited capacity for repair after injury ${ }^{5,6}$. Therefore, bone marrow-derived MSCs are attractive candidates in therapeutic agents for neurological diseases because of their relative ease in harvesting, isolation, expansion and multi-differentiation ${ }^{7}$. The growing interest in MSCs has led to a number of studies in which their differentiation properties have been examined ${ }^{8,9}$, during the last several years, there have been many reports describing in vitro neural transdifferentiation of MSCs derived from a range of different species ${ }^{10,11}$, but, despite the fact that neuronal-like morphology and neuronal proteins expression under culture or in vivo conditions have been observed, until now neuronal functional properties have not been demonstrated and whether MSCs can actually differentiate into neurons remains controversial ${ }^{12}$.

As we know, that neurons developed from embryonic stem cells, needs many genes, factors and signal control, which the transcriptional factor Nurr1 was first discovered by Law et al. ${ }^{13}$ and was found to be a critical role for Nurr1 in the development of dopamine neurons ${ }^{14}$. Subsequent studies confirm that Nurr1 is involved in regulating the differentiation, migration and maturation of mesencephalic dopaminergic neurons and controls the expression of key genes involved in dopamine 
(DA) neurotransmission, e.g., tyrosine hydroxylase (TH) and the dopamine transporter (DAT) (15). Transcripts for achaetescute complex homolog 1 (ASCL1, also known as Mash1), a transcription factor that plays a role in neural differentiation during embryogenesis and in the generation of olfactory and autonomic neurons ${ }^{16,17}$, too. Mash1 is expressed, during the development of central nervous system and peripheral nervous system and Mash1 has a very important role on neuronal cell differentiation. But we do not know its exact mechanism, therefore, further research of Nurr1 and Mash1, especially studies about their regulatory mechanisms on neuron differentiation have potential clinical application.

In view of the potential therapeutic application of MSCs for neurodegenerative diseases, it has become essential to examine whether neural-induced MSCs display the functional characteristics of neural cells. For this, in the present study, we evaluated the multipotent capacity of MSCs and investigated whether MSCs could be directed to differentiate into dopaminergic neurons in vitro by forced expression of the transcription factor Nurr1 and Mash1 and attempted to investigate the neurotransmitter phenotype displayed by the MSC-derived neurons generated from our protocol and to evaluate the usefulness of Nurr1 and Mash1 for monitoring of cellar differentiation by measuring the change of the cellar functional hallmark of neurons, including the expression of mesengenic differentiation markers (neuronal-like morphology and neural functional hallmark).

\section{EXPERIMENTAL}

Cell surface antigen expression of CD11b (Invitrogen, Carlsbad, USA), CD14 (eBiosciences, San Diego, CA, USA), CD34, CD45 (Beckman Coulter, Villepinte, France), CD73, CD90 and CD105 (BD Biosciences, Le Pont de Claix, France), CD166-FITC (AbD Serotec, Cergy St Christophe, France) and HLA-DR-CY5 (Abcam, Cambridge, UK) was measured by flow cytometry.

Immunofluorescent staining of cryosections was then performed as described ${ }^{28}$. Antibodies used were: anti-pairedlike homeodomain transcription factor 3 (Pitx3, 1:1000), antidopamine transporter (DAT, 1:800) and antiNurr1 (1:500, E-20 sc-990 X, all from Santa Cruz Biotechnology Inc., Milan, Italy); anti-Lamx1a (1:1,000; gift from Mike German, University of California, San Francisco, CA), anti-Ngn2 (1:50; Santa Cruz Biotechnology, Santa Cruz, CA, USA), anti Mash1 (1:500, BD Biosciences, Heidelberg, Germany), anti En2 (1:25; DSHB, Iowa, USA); Neuron Specific Enolase (NSE, 1:400) and antineurofilament medium 160KD (NF-M, 1:50, Abcam, Cambridge, MA, US), antimicrotubule associated protein-2 (MAP2, 1:200) and antineuron-specific class $\beta$-III tubulin (Tuj1, 1:1 000) and Neuronal Nuclei (NeuN, 1:500; from Covance, Princeton, NJ, USA), rabbit antinestin (1:200) and antihuman nuclei (NeuN, 1:500; from Chemicon, Temecula, CA, USA); anti Tau protein (1:50) and antiglial fibrillary acidic protein (GFAP, 1:50, all from DAKO Corp., Carpinteria, California, USA); tyrosine hydroxylase (TH, 1:1 000, SigmaAldrich, St. Louis, MO, USA) and GAPDH (1:500; Acris Antibodies GmbH, Hiddenhausen, Germany) as monoclonal antibodies. Primary antibodies were incubated overnight at
$4{ }^{\circ} \mathrm{C}$ and primary antibodies were detected by using FITC- or Cy3-conjugated goat anti-rabbit or anti-mouse secondary antibodies (1:200, Sigma, USA) for $1 \mathrm{~h}$ at room temperature. After reaction with secondary antibodies, the cells were stained with 100 nM DAPI (Sigma, USA) for 15 s, then mounted. Fluorescence-labeled MSCs were viewed and photographed (AX10, ZEISS, German). Immunoblots were incubated with goat anti-rabbit (1:500, Bio-Rad) or antimouse IgG antibodies (1:500, Amersham) and the reaction detected with the ECL plus procedure (Amersham).

All restriction enzymes and T4 DNA ligase were purchased from New England Biolabs (USA); CIP (Alkaline Phosphatase, Calf Intestinal) enzyme, Endo-Free Plasmid Maxi Kit (\#D6926-B) and E.Z.N.A. Gel Extraction Kit (\#D2500, D2501) were purchased from omega (USA); DH5 $\beta$ bacteria and adenovirus pAdxsi vector were purchased from SinoGenoMax (China). The pUC57-Nurr1 and pUC57-Mash1 plasmids were generated and saved in our institute. Lipofectamine 2000 was from Invitrogen (USA). The primers were synthesized by Boya Corp, Shanghai (China). RT-PCR kit was purchased from TAKARA Biotechnology (Dalian) CO., Ltd. DMEM/F12 medium and B27 were purchased from GibcoInvitrogen (USA). Fetal bovine serum was from Hyclone (USA).

Culture of bone marrow mesenchymal stem cells (MSCs): Human bone marrow of five healthy donors aged 21 to 25 years was obtained from Institute of cell therapy center, after they gave informed consent according to the approved procedure. Procedures were approved by the Local Ethical Committee for Clinical Research in Anhui university of science $\&$ technology. About 8-12 mL of marrow were collected from the pelvis of each donor and stored in a heparin containing test tube. After Ficoll-Plaque (density of $1.077 \mathrm{~g} \mathrm{~mL}^{-1}$ ) purification of whole bone marrow, mononuclear cells were isolated and seeded into polystyrene plastic $75 \mathrm{~cm}^{2}$ tissue culture flasks at $37{ }^{\circ} \mathrm{C}$ in $5 \%(\mathrm{v} / \mathrm{v})$ humidified $\mathrm{CO}_{2}$ at a density of $2 \times 10^{5}$ cells $\mathrm{cm}^{-2}$ in DMEM supplemented with $15-20 \%(\mathrm{v} / \mathrm{v})$ fetal bovine serum (Gibco). After $24 \mathrm{~h}$, non-adherent cells were removed, Adherent cells, namely, MSCs, were subjected to subculture when they reached $95 \%$ confluence and were subcultured 4 times. The purity of MSCs was over $95 \%$ after 20 days from initial seeding. The MSCs used in the present study were from passages 4 to 5 .

Characterization of bone marrow mesenchymal stem cells: Bone marrow mesenchymal stem cells were harvested in trypsin containing ethylenediaminetetraacetic acid (hyclone), washed twice with phosphate-buffered saline (PBS) and stained for $45 \mathrm{~min}$ at room temperature according to the manufacturer's instructions with monoclonal antibodies (BD Biosciences PharMingen $^{\mathrm{TM}}$, Heidelberg, Germany), including HLA-DR, CD11b-FITC, CD14-FITC, CD34-FITC, CD44-PE, CD45FITC, CD73-PE, CD90-PE, CD105-FITC, CD166-PE and control (IgG1-FITC and IgG1-PE, BD). The experiment was made in triplicates $(n=3)$ and 30,000 events were registered for each sample, then MSCs were characterized by flow cytometry (BD FACSCalibur System, BD Biosciences Immunocytometry System, Heidelberg, Germany) and their differentiation potential was investigated. Adipogenic and osteogenic differentiation in vitro was monitored as previously described ${ }^{18}$. Lipid vacuoles 
and calcium deposits were observed and identified by Oil Red O (Sigma) and Von Kossa staining methods, respectively, specific operation see References ${ }^{19}$. In particular, in this study, MSCs immunophenotype and differentiation capacity were evaluated at late passages as P4 and P10.

Construction of Adxsi-Nurr1-Mash1 virus vector: To co-express Mash1 and Nurr1, pAdxsi-Nurr1-Mash1 was constructed using pShuttle-GFP (Invitrogen Carlsbad, CA), with cloning of Mash1 behind the CMV1 promoter and that of Nurr1 behind the CMV2 promoter. The Mash1 gene fragment was retrieved from pUC57-Mash1 vector by BglII and EcoRI double-enzyme digestion and pShuttle-GFP plasmid was digested with the same enzymes and separated by gel fractionation. then purified using E.Z.N.A gel extraction kit (Omega Bio-tek, Inc, GA, USA). Next, ligation of Mash1 fragment at gene to vector molar ratios of 1:3 using T4 DNA ligase at $16^{\circ} \mathrm{C}$ overnight. Following transformation in DH5 $\beta$ competent cells and amplification in terrific broth media, plasmids were purified using GenElute ${ }^{\mathrm{TM}}$ Plasmid Miniprep kit (Sigma, St. Louis, MO) and evaluated pShuttle-GFP-Mash1 by double restriction digestion using BgIII and EcoRI enzymes, for single copy gene insertion in the right orientation. Nurr1 cDNA was excised from pUC57-Nurr1 vector using Xho I restriction digestion to linearized and purified as described above. Then digested with enzyme klenow for $24 \mathrm{~h}$ and purified as described above. Next the product pUC57-Nurr1 fragment was digested with Nhe I and purified as described above. pShuttle-GFP-Mash1 vector was digested by Nhe I and Pme I double-enzyme and then purified as described above. The Nurr1 cDNA was ligated with the linearized pShuttle-GFPMash1 vector at the gene to vector molar ratios of $1: 3$ and using T4 DNA ligase for $16 \mathrm{~h}$ at $16^{\circ} \mathrm{C}$. Following transformation of the ligated mixture in DH5 $\beta$ competent cells and amplification in terrific broth media, the resulting plasmid, pShuttle-Nurr1-Mash1, was purified and confirmed by restriction digestion using Nhe I and Pme I enzyme. Plasmid was characterized in terms of concentration, purity and integrity by UV spectrophotometry at 260/280 $\mathrm{nm}$ and agarose gel electrophoresis.

For the construction of pAdxsi-Nurr1-Mash1 plasmid, pAdxsi plasmid and pShuttle-Nurr1-Mash1 plasmid were digested with I-Ceu I and I-Sce I enzymes and then dephosphorylated, which were purified as described above. The DNA ligation was carried out by incubating in the $15 \mu \mathrm{L}$ reaction solution containing $1.25 \mu \mathrm{L}(80 \mathrm{ng} / \mu \mathrm{L})$ of the digested pAdxsi plasmid and pShuttle-Nurr1-Mash 1 at $16{ }^{\circ} \mathrm{C}$ overnight, before being ligated with the inserted fragment of interest to generate the pAdxsi-Nurr1-Mash1 viral plasmid. This plasmid was also amplified in DH5 $\beta$ cells. Plasmid was characterized in terms of concentration, purity and integrity by UV spectrophotometry at 260/280 nm and agarose gel electrophoresis.

Virus production and titer determination: pAdxsiNurr1-Mash1, which was digested with Pac I enzyme, transfectedinto HEK 293 cells with Lipofectamine 2000. The cell supernatant containing a large number of viruses was collected 3-5 days after transfection when most of the cells appeared plaques. The titer of virus was determined using 293 cells following the AdEasy Vector Systerm method while an increase in titer was found after profelication in HEK293 cells.
Neuronal induction: After 5 passages, when cells are positive for mesenchymal markers (CD73, CD90, CD105, CD166) and negative for hematopoietic/endothelial markers (HLA-DR, CD11b, CD14, CD34 and CD45) (data not shown), About $80 \%$ confluent MSC cultures were exposed to the infectious Adxsi-Nurr1-Mash1 virus at a multiplicity of infection (MOI) of $1.0 \times 10^{2} \mathrm{pfu} /$ cell in DMEM medium for $1 \mathrm{~h}$, the medium was then removed and then Adxsi-Nurr1-Mash1 transfected MSCs (Nurr1/Mash1-MSCs) were cultured in Neurobasal-A medium (Invitrogen) supplemented with $10 \%$ (v/v) B27 supplement (Gibco), 5 $\mu$ M GSK-3 inhibitors (TWS119, Sigma) and $25 \mathrm{ng} / \mathrm{mL}$ nerve growth factor (NGF, Sigma). Medium was changed every $48 \mathrm{~h}$ subsequently. At the end of the cultivation period, the cells were photographed and then fixed with frozen pyruvate for $10 \mathrm{~min}$, preparing for the immunological assay and Western Blot assay. The cells were also used for RNA extraction and reverse transcriptionpolymerase chain reaction (RT-PCR) analysis of neurogenic gene expression.

Immunological assay: Uninduced and induced MSCs were established on glass coverslips placed in 35-mm culture dishes. On the day of immunofluoresence labeling, cells were washed with PBS ( $\mathrm{pH} 7.4$ ) and then fixed with $4 \%$ (w/v) paraformaldehyde for $10 \mathrm{~min}$ and treated for $10 \mathrm{~min}$ with 0.1 M glycine to quench autofluorescence and followed by permeabilization in $1 \%$ Triton-X. Cells were incubated overnight at $4{ }^{\circ} \mathrm{C}$ with the following antibodies: primary antibodies against GFAP, $\beta$-tubulin III, Nestin, NSE, NF-M, MAP2 and TH. The following day, cells were rinsed with washing buffer (PBS plus $0.3 \%$ Triton $\mathrm{X}-100$ ) and incubated at room temperature for $1 \mathrm{~h}$ in the dark with appropriate fluorochrome-conjugated secondary antibodies (1:200, Sigma, USA). Primary antibodies and secondary antibodies were diluted in $0.1 \%$ bovine serum albumin (BSA)/PBS. Primary antibodies were developed with secondary FITC-goat antimouse and PE-goat antirabbit, at final concentrations of 1/500. Cells labeled solely with PE- and FITC-IgG served as isotype controls. Following labeling, Nuclei were counterstained with 300 nM DAPI. Cover slips were immediately transferred to glass coverslides and analyzed as described in the immunocytochemistry method.

Gene expression analysis: Total RNA was extracted with trizol (Invitrogen, USA) according to the manufacturer's instructions cDNA was synthesized from mRNA using the AMV reverse transcriptase (Gibco, USA) and 200 ng of cDNA was used in PCR to amplify goal genes. Relative abundance of mRNA was calculated after normalization to GAPDH ribosomal RNA. The following primers were manufactured by Sigma, UK (Table S1) and used for real-time PCR. PCR reactions were normalized by amplifying the same sample of cDNA with primers specific for glyceraldehyde-3-phosphate dehydrogenase, GAPDH. Thermocycling conditions were $30 \mathrm{~s}$ at $95^{\circ} \mathrm{C}, 30 \mathrm{~s}$ at $54-65^{\circ} \mathrm{C}$ and $30 \mathrm{~s}$ at $72^{\circ} \mathrm{C}$ for $28-30$ cycles preceded by $10 \mathrm{~min}$ at $95^{\circ} \mathrm{C}$, final extension at $72^{\circ} \mathrm{C}$ for $10 \mathrm{~min}$ (optimized per primer set as described in Supplementary information Table-1). RT-PCR products $(10 \mu \mathrm{L})$ were separated by electrophoresis on $2 \%$ agarose gels (Sigma Chemical Co.) and visualized with $0.5 \mu \mathrm{g} / \mathrm{mL}$ ethidium bromide. Band sizes were compared with $1 \mathrm{~kb}$ DNA ladder (Invitrogen, Carlsbad, CA). 
Immunoblotting analysis: Nucleus or cytoplasmic protein extract $(20 \mu \mathrm{g})$ from induced MSCs or uninduced MSCs were electrophoretically separated using $10 \%$ SDS-PAGE pre-cast gel (Invitrogen) and transferred onto a nitrocellulose membrane (GE HealthCare, England). Then, membrane blocking, washing and antibody incubation were carried out according to the manufacturer's instructions. The blot was probed for Antibodies against Nurr1, Mash1, Ngn2, Pitx3, En2, Lmx1a, NF-M, $\beta$-III tubulin and GFAP were used. Anti GAPDH and anti PRPP immunoblotting analysis was performed as a loading control. Membrane was then washed and incubated with appropriate horseradish peroxidas-conjugated secondary antibodies and detected using an ECL chemiluminescence system (GE HealthCare, England). Immunoprecipitants (40 $\mu \mathrm{L})$ were assayed for total protein concentration and then analyzed by western analysis.

Quantification of tissue dopamine (DA) levels: We used a previously published method to quantify tissue dopamine levels ${ }^{20}$. Medium was removed from the wells of the 24-well plate at $1.5 \times 10^{5}$ cells/well and replaced with $100 \mu \mathrm{L}$ of $0.1 \mathrm{M}$ $\mathrm{HClO}_{4}$ solution containing $7.9 \mathrm{mM}$ sodium metabisulfite, 1.3 $\mathrm{mM}$ EDTA and $10 \mathrm{nM}$ dihydroxybenzylamine (DHBA) as an internal standard. The cells were removed by scrapping and the wells were washed with an additional $100 \mu \mathrm{L} \mathrm{HClO}_{4}$ solution. Samples were assayed individually. Cellular suspensions $(200 \mu \mathrm{L})$ were sonicated and centrifuged for $15 \mathrm{~min}$ at 13000 $\times$ g at $4{ }^{\circ} \mathrm{C}$. Samples were filtered $(0.22 \mu \mathrm{m})$ and $50 \mu \mathrm{L}$ aliquots were subjected to HPLC analysis. Specific operation reference manual and all chemicals were purchased from Sigma-Aldrich, St Louis, MO, USA.

Ethical statement: Usages of human specimens in this study were approved by the Ethics Committee of Anhui University of Science \& technology, China. under protocol 2010020.

Statistical analysis: Protein and mRNA levels were quantified by measuring the optical density of each band using computer-assisted densitometry (NIH Image analysis program, version 1.61). Data were evaluated for statistical significance using SPSS 12.0. Values are expressed as the mean \pm SEM. The one-way analysis of variance (ANOVA) test (Scheffe's multiple comparison) was used to analyze differences between groups, with $p<0.05$ being considered significant.

\section{RESULTS AND DISCUSSION}

Purification and characterization of MSCs: MSCs are isolated from bone marrow by their adherence to plastic ${ }^{21}$. We eliminate myelopoietic cells that express the cell surface receptor $\mathrm{CD} 11 \mathrm{~b}$ from the cultures via immunodepletion using an anti CD11b antibody. After immunodepletion, untreated MSCs appear as a uniform population of fibroblastic-shaped cells (Fig. 1A) whose characteristic feature is a CD11b ${ }^{-}, \mathrm{CD}^{-} 5^{-}$,

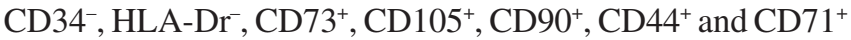
cell surface phenotype, consistent with previous reports ${ }^{22}$. All these antigens were confirmed by flow cytometry. Before neurogenic induction, MSCs were also evaluated for their multilineage capacity. These cells can be induced to differentiate into osteocytes and adipocytes lineage using specific culture media (Fig. 1B, 1C).
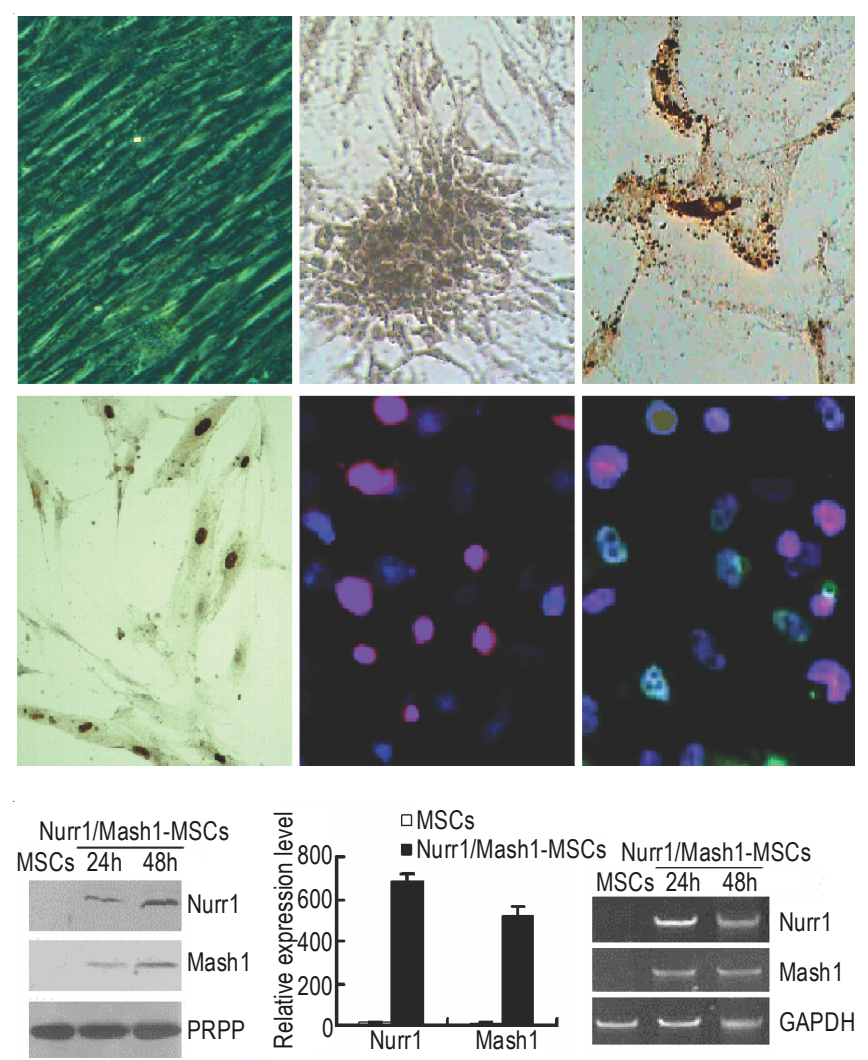

Fig. 1. Mormal morphology and pluripotency of MSCs and target genes expression in Nurr1/Mash1-MSCs. (A) MSCs displayed an homogeneous morphology of fibroblastic cells (400x); (B) Under specific induction MSC were differentiated intoadipocytes (lipid vacuoles were coloured by oil red O, (400x); (C) osteocytes (calcium deposits were revealed by Von Kossa method, (400x); (D) neuronlike cells derived from MSC upon treatment with neurogenic medium (400x); (E and F) Expression of nestin and MAP2 after 10 days induction, detected by immunofluorescence $(400 \times)$; $(\mathrm{G})$ Analysis of Nurr1 and Mash1 expression in Nurr1/Mash1-MSCs. (H) WB analysis of Nurr1 and Mash1 expression in Nurr1/Mash1MSCs (left panel); (H) RT-PCR analysis of Nurr1 and Mash1 relative gene expression in MSCs and Nurr1/Mash1-MSCs (middle panel) and the right panel shows RT-PCR analysis of the gene of Nurr1 and Mash1 (right panel). PCR products were electrophoresed in an agarose gel and stained with EB. Error bars represent SEM, n = 3

AS Nurr1 sufficiently induces the acquisition of dopamine neuron phenotype in the cultured neural precursor cells isolated from rat embryonic brains and a specific role of Mash1 in the development of the midbrain dopamine neurons ${ }^{23}$, we examined the effect of co-expressing Mash1 with Nurr1 in MSCs. Levels of Nurr1 and Mash1 in the supernatant of cultured MSCs and Nurr1/Mash1-MSCs were studied. Nurr1/Mash1-MSCs stably expressed Nurr1 with Mash1 after transfected 24 h. Nurr1/ Mash1-MSCs showed similar spindle-shaped morphology. IHC (Fig. 1D), IFA (Fig. 1E, 1F), WB (Fig. 1G) and PCR (Fig. 1H) analysis of the Nurr1/Mash1-MSCs was essentially identical to primary MSCs. Non-transfected MSC did not produce Nurr1 or Mash1 transcription factor.

Neuronal like differentiation of Nurr1/Mash1-MSCs: The Nurrl-expression in ES cells can lead to up-regulation of several dopamine neuroal markers expression, which suggests Nurrl is a critical for the development of dopaminergic (DA) neurons ${ }^{24}$. On the other hand, Mash1 appeard to be critical important, as leaving it out abolished any positive staining for 
Tuj1 staining ${ }^{25}$. So, we examined the effect of co-expressing Mash1 with Nurr1 in MSCs. The Adxsi-Nurr1-Mash1 vector system showed a consistent transduction efficiency of $>85 \%$ for all cells as examined by immunocytochemical analysis of transgene expression (Fig. 2A). Some cellular cytoplasm gathered towards the nucleus and formed the axons and dendrites (Fig. 2B,C) and significant morphological transformation into neuron-like cells occurred 2-3 days after gene transduction. Forced expression of Mash1 and Nurr1 resulted in extensive neurite outgrowth from Mash1/Nurr1-MSCs and expression of neuron-specific markers (Nestin, NSE) (Fig. 2B,C). The majority of Nurr1/Mash1-MSCs transformed into neuron-like cells, they had large and round cell bodies with longer axons and part of cells became simple bipolar or complex multipolar with highly branched processes with intense NSE staining is evident in both neuronal cell types.
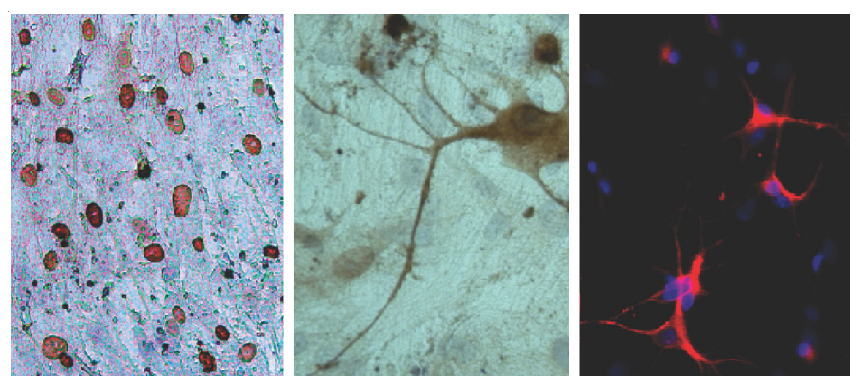

Fig. 2. Transcription factor locates, morphological changes and neural markers expresss in Nurr1/Mash1-MSCs after transfection of 2factors. (A) Transcription factor Mash1 locates in the nucleus of Nurr1/Mash1-MSCs(400x); 48-72 h after induction, some cellular cytoplasm gathered towards the nucleus and formed the axons and dendrites and began to expressed neuronal related molecule NSE (B) (400x) and Nestin ( C) (200x)

Overexpression of Nurr1 and Mash1 up-regulates Neural-related transcription factors $\mathrm{mRNA}$ and protein: Transcription factors required for neuron development. Mash1 and Nurr1 which play a role in neuronal differentiation and late differentiation of dopaminergic neurons ${ }^{26}$. As Ngn2 was required for neuronal differentiation and mainly expressed in progenitors and immature neurons ${ }^{27,28}$, Pitx3, Lmx1a and En1/ $2^{29-31}$ were required for maintenance of mature mDA neurons and survival of mature mDA neurons, which can express in progenitors, immature and mature neurons. We subsequently focused on neural markers expression in differentiating cells. So, we examine neural-related transcription factors $\mathrm{Ngn} 3$, Lmx1a, En2 and Ptx3 etc. expression in neuronal like differentiating cells induced by Nurr1 and Mash1 interval different time up to eight days, the expression was analyzed in Western blot and the results were normalized to the expression of PRPP (Fig. 3). Lmx1a expression was only weakly detected during the first two days in vitro, yet it increased progressively. The pattern of $\mathrm{Ngn} 2$ expression closely resembled that observed for Lmx1a. Pitx 3 expression was hardly detected during the first two days in Adxsi-Nurr1Mash1 transfected MSCs in vitro, yet it was only weakly detected four days later and it increased progressively thereafter. By contrast, En2 expression was clearly evident from the first two days and its expression was sustain and stable.
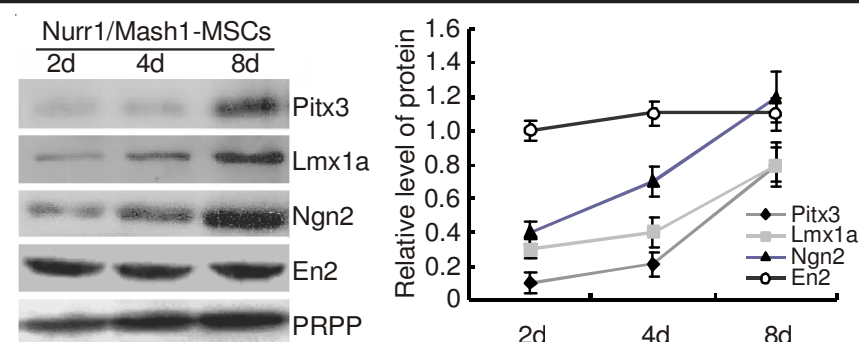

Fig. 3. Neural-related transcription factors expression in neuronal like differentiating MSCs induced by Nurr1 and Mash1. Western Blot analysis for neural related transcription factors in differentiated MSCs. (A) Differentiated cells express neuronal related transcription factors Ngn3, Lmx1a, Ngn3, En2 and Ptx3; (B) At different times after cell transfectiont, related transcription factors Ngn3, Lmx1a, Ngn3, En2 and Ptx3 expression levels were evaluated for AdxsiNurr1/Mash1-induced MSCs. Error bars represent SEM, n = 3

Neural markers expression in neuron-like differentiated cells: To characterize neuronal differentiation further we fixed neuron-like differentiated cells transfected by Nurr1 and Mash1 for 8 days, we stained differentiated cells and MSCs for the neuronal early cytoplasmic nonspecific marker neuronspecific enolase (NSE), respectively. Unresponsive, typical, flat MSCs expressed very low and were only lightly stained, consistent with their undifferentiated state. Western blot analysis also confirmed the expression of low levels of NSE protein in uninduced MSCs, but detectable, NSE stably expressed in neural-like cells. Nurr1/Mash1-MSCs-derived neural-like differentiated cells displayed distinct neuronal morphologies (Fig. 4A), ranging from simple bipolar to large, extensively branched multipolar cells (Fig. 4A). AS tau protein is mainly located at the cytoplasm of neurons where it facilitates tubulin assembly into microtubules, stabilizes polymerized microtubules and promotes the axonal formation of cytoplasmic extensions $^{32}$; the appearance of tau is improtant for neuron development and it is considered a early neuronal marker of neuron development ${ }^{33,34}$, we found that neuron-like differentiated cells formed axons-like cytoplasmic extensions and tau protein strongly detected in neuron-like differentiated cells (Fig. 4B). As tau is expressed with other microtubule associated proteins (for example dendritic marker MAP2) during neuron development ${ }^{35}$. Microtubule-associated protein 2 (MAP2), also an early neuronal marker and playing an important cytoskeletal role in development and function of the nervous system ${ }^{36}$, was strongly detected in neuron-like differentiated cells (Fig. 4C), whereas no more than $0.1 \%$ of MSCs were positive to MAP2 in the control MSCs cultures. Other widely used markers for neurons are neurofilament-medium (NF-M), which is expressed early during neurogenesis and plays many roles in neurite outgrowth, including protein trafficking, cellular motility and maintaining neurite structure ${ }^{37}$, was specifically up-regulated expression in neuron-like differentiated cells (Fig. 4D) and remained elevated at 8 days. Tyrosine hydroxylase $(\mathrm{TH})$, a neurotransmitter-related enzyme in catecholaminergic neurons, was also significantly detectable (Fig. 4E). To investigate neuronal characteristics further, we stained differentiated cultures for Neu N, a neuron-specific marker ${ }^{38}$. Induction of the neuronal phenotype resulted in a dramatic increase in $\mathrm{Neu}$ $\mathrm{N}$ expression (Fig. 4F), too, which suggest induced neuronlike cells may have biological properties of neurons. 

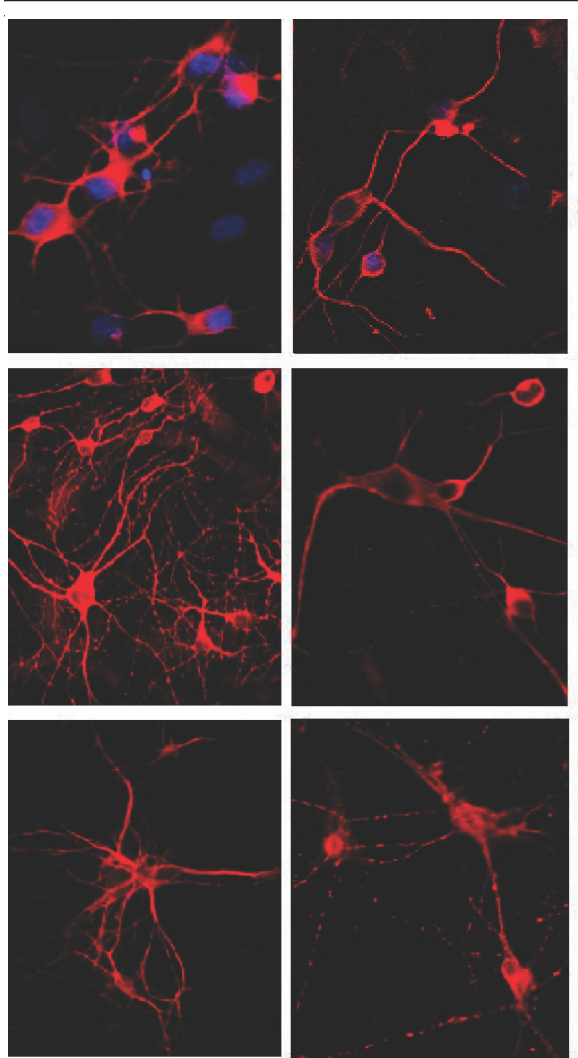

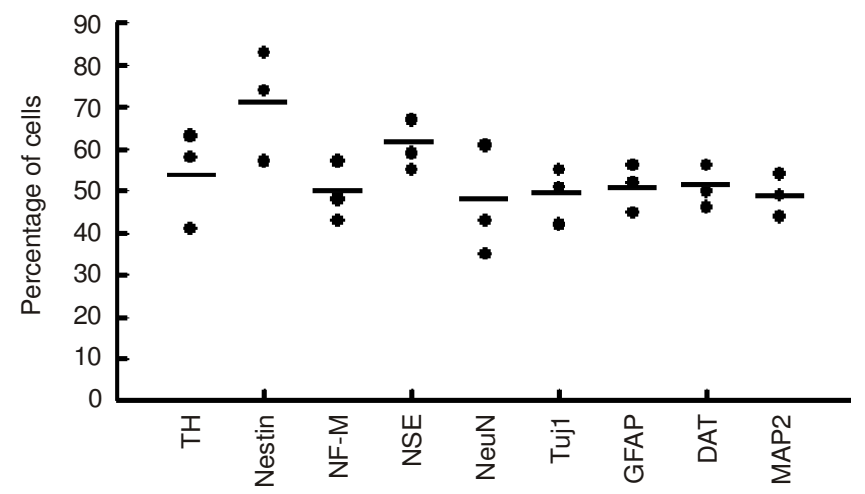

Fig. 4. Analysis of neuron cell marker expression. Immunofluorescence of neuron-like cells after 8 days of Adxsi-Murr1-Mash1 transfection, neuron-like cells display condensed cell bodies, highly branched processes including simple bipolar cells or complex multipolar and exhibit neuronal morphologies, the Neuron-like cells were immunoreactive for the neuronal markers (A) Neuron specific enolase (NSE), (B) tau protein, (C) microtubule-associated protein 2 (MAP2), that is suggested to play an important cytoskeletal role in development and function of the nervous system, (D) neurofilament medium (NF-M), major intermediate filaments found in neurons, comprise the axoskeleton and functionally maintain neuronal caliber and also play a role in intracellular transport to axons and dendrites, (E) tyrosine hydroxylase (TH), (F) Neu N, (G) $\beta$-III Tubulin, (H) Nestin and (I) Nestin(red) and DAT (green) were detected in neuron-like cells (400x). (J) Quantification of the percentage of stained immunofluorescence for neural related proteins positive cells from Nurr1/Mash1-MSCs revealed following data: NSE expression was found in $60.3 \pm 12.4 \%$ of all cells, $54 \pm 18.2 \% \mathrm{TH}$ positiv e cells, $51 \pm 16.2 \%$ of the cells expressed GFAP, $50.7 \pm 15.8 \%$ of the cells expressed DAT, $49.3 \pm 15.5$ and $49.3 \pm 17.1 \%$, of the cells expressed Tuj1 and NF-M, respectively, only $46 \pm 12.9 \%$ of the cells expressed Neu N. Nestin expression was found to be present in all cells analyzed. in $71.3 \pm 13.1 \%$ of all cells. (K) Results of RT-PCR in Neuron-like cells. Neuron-like cells contained high levels of TH, nestin, NF-M, NSE and Neu N mRNA. (L) Western blot analysis confirms expression of TH, nestin, NSE, NF-M, Tuj1, DAT and MAP2. Before neuronal differentiation, MSCs lack of neuronal markers expression

We further examined neurons moleculal markers Nestin, $\beta$-III tubulin (Tuj1) and DAT in neuron-like cells derived Nurr1/Mash1-MSCs. Very encouraging, neuron-like cells stained positive for the general neuron-specific marker Tuj1 and other markers more specifically expressed in dopamine neurons (Fig. 4G, 4H, 4I), which included Tuj1, Nestin and dopamine transporter (DAT).

Moreover, quantification of the percentage of stained immunofluorescence for neural related proteins positive cells from neuron-like differentiated cells (Fig. 4J). In addition, these neuron-like cells stained negative for serotonin and ChAT (markers for serotogenic neurons and cholinergic neurons, respectively). We next determined the expression levels of several dopamine neuron-specific genes through RT-PCR in the neuron-like cells (Fig. 4K). Results showed clear and evaluated expression of dopamine neuron-specific genes TH, nestin, NF-M, NSE and Neu N. The expression levels of these genes increased tremendously in the neuron-like cells when compared with control MSCs (Fig. 4K). In fact, there was minimal expression of these genes in MSCs. We also examined the expression of neuron-specific protein TH, nestin, NSE, NF-M, Tuj1, DAT and MAP2 through WB(Figure 4L). Our results indicated that strong expression of $\mathrm{TH}$, nestin, NSE, NF-M, Tuj1, DAT and MAP2 neuron-specific protein could be detected in differentiated neuron-like cells and expression of Tuj1, NSE, NF-M, DAT and MAP2 were strongly induced and all was found in more than $45 \%$ of neuron-like cells, which in agreement with the higher abundance of neuronspecific markers mRNA and protein measured in these cells.

Dopamine release in response to membrane depolarization: Forced expression of Mash1 and Nurrl resulted in extensive neurite outgrowth from Nurr1 and Mash 1 positive cells with significant alterations in the number of cells positive for $\mathrm{TH}$ protein expression, a late mature dopamine neuronal marker. While very encouraging, these results did not confirm the functionality of the dopamine neuron-like cells. So, we next evaluated mature dopamine neuronal functions of the cells transfected with Adxsi-Murr1-Mash1 by estimating dopamine 
release and specific dopamine uptake in these cells, because specific, DAT-mediated dopamine uptake is one of the key characteristics of dopamine neurons. HPLC analysis demonstrated that dopamine levels in the medium conditioned for $24 \mathrm{~h}$ was about $217.6 \pm 35.2 \mathrm{pg} / \mathrm{mL}$ in the dopamine neuron-like cells culture, which was significantly higher than that in MSCs culture (33.6 $\pm 21.3 \mathrm{pg} / \mathrm{mL})$ (Fig. 5). One of the most important functional aspects of mature dopamine neurons is the ability to release dopamine in response to membrane depolarization. When cells in the medium only for $0.5 \mathrm{~h}$, both dopamine neuronlike cells and MSCs hardly secret detectable levels of dopamine by HPLC. However, treatment with $56 \mathrm{mM} \mathrm{KCl}$ induced dopamine release from dopamine neuron-like cells. The dopamine levels evoked by KCl-depolarization stimuli for $10 \mathrm{~min}$ were fivefold and for $15 \mathrm{~min}$ were eightfold higher in the cultures of dopamine neuron-like cells $(547.2 \pm 64.6$ and $856.5 \pm 95.1 \mathrm{pg} / \mathrm{mL}$, respectively) (Fig. 5). Moreover, only dopamine neuron-like cells for more than 20 days showed specific dopamine uptake, indicating that the dopamine neuron-like cells possess dopamine neurons biological properties.

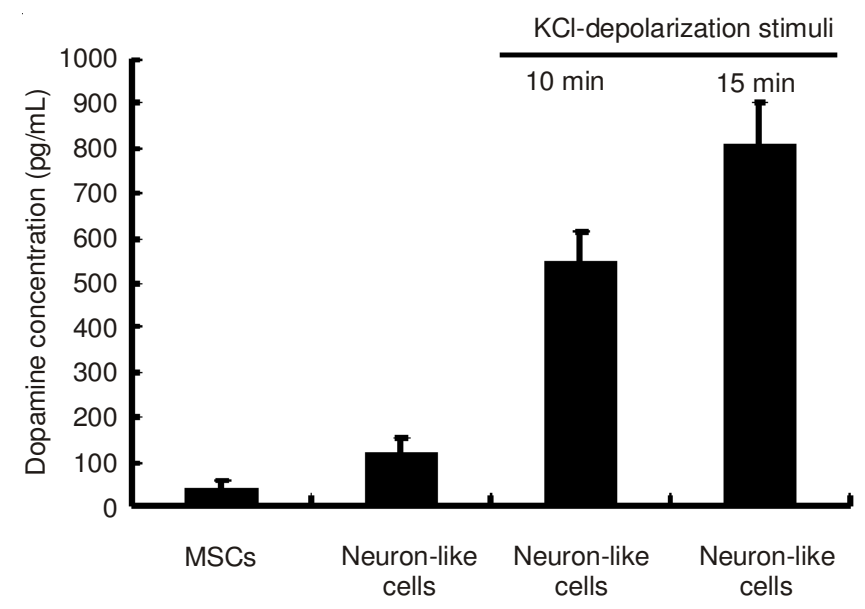

Fig. 5. Dopamine release and uptake of neuron-like cells. quantitative results of HPLC chromatography experiments measuring dopamine from dopamine-neuron like cells. Error bars represent SEM, n = 3

Recent observations demonstrated the capacity of MSCs to differentiate into neuron tissue. However, molecular mechanisms implied in the differentiation process remain largely unknown. A great number of studies have evaluated the neuronal differentiation potential of MSCs in vitro, using different chemical agents protocols of induction ${ }^{37-39}$, but genes modulated by chemical agents induction were different from those observed during neurogenesis in neural tissue ${ }^{27,29,33}$, suggesting that the rapidity of neuron-like differentiation argues against physiological cell differentiation.

In this study, we developed the method of transfected key transcription factors, through cascades of transcriptional events, to induce into neuron-like cells from genetically engineered MSCs. MSCs ectopically expressing Nurr1 and Mash1 have a significantly increased neuron-specific markers expression and dopamine response. During the differentiation process and contrasting to previous studies, we did not observe morphological begin to change after 2 days using our protocol.
An average of four days was required to display cells with neuron-like morphologic characteristics and 7-8 days to stably obtain more than $50 \%$ of differentiated MSCs. Removing the neurogenic medium the differentiated cells did not spontaneously return to their fibroblastic morphology within the three weeks. Due to stability of the differentiated state, to understand well the molecular basis for the transdifferent effect of Nurr1/ Mash1 in MSCs, we analyzed the profile of gene expression during the differentiating process, including transcription factors Pitx3, Lmx1a, Ngn2 and En2; neuron markers Tau, TH, nestin,MAP2, GFAP, NF-M and $\beta$-III Tubulin. By RT$\mathrm{PCR}$ and $\mathrm{WB}$, we compared expression levels of several neuron specific transcription factors including transcription factors Pitx3, Lmx1a, Ngn2 and EN2 expression level difference between neuron-like cells and MSCs. Analysing these results, we observed that neuronal specific genes (En2, Lmx1a, Pitx3, Mash1 and Nurr1) were upregulated during the neurogenic differentiation of MSCs ${ }^{23,24,26,38}$ and neuron-like cells were positive to TH, MAP2, NSE, MAP2, DAT, Neu N, NF-M and $\beta$-III Tubulin, but continued to express the neural stem cell marker nestin. Interestingly, we also observed that not only genes related to neurogenesis were increased in neuron-like cells, but also neuron-like cells released dopamine in response to a depolarizing stimulus in vitro. Indeed, each gene taken separately, we cannot conclude that MSCs can differentiate into neurons, but these findings assembling all upregulated/ down regulated genes and dopamine in response, the differentiation potential of Nurr1 with Mash1-engineered MSCs transforming into neural cell lineage seems to be confirmed. Taken together, we speculate that Nurr1 and Mash1 with neurogenic medium can start cascades of transcriptional events, push transcription factors and neuron decisive genes temporal expression and activate the neuronal differentiation pathway. Nevertheless, persistent high expression of $\mathrm{TH}$, MAP2, NSE, MAP2, DAT, Neu N, NF-M and $\beta$-III Tubulin after 7 days of differentiation and stable neuronal cell morphology with the expression of functional dopamine molecule suggests Nurr1 and Mash1 modification endows MSCs capable of transforming into neuron ${ }^{15,16,39}$.

Nevertheless, our results confirm that in vitro Nurr1 with Mash1 inducing differentiation protocol could be sufficient to differentiate MSCs into neuron-like cells. Further studies are required to compare and analyze the characteristics of neuron-like cells originating from Adxsi-Nurr1-Mash1 transfected MSCs in molecular biological and physiological aspects and confirm whether these neuron-like cells can be possibly integrated into pre-existing neural networks in the injured spinal cord slice while maintaining expression of neuronal and MN-specific markers.

In summary, we demonstrate that Nurr1/Mash1-derived MSCs could be induced and transdifferentiated into functional neuron-like cells in the appropriate culture conditions. These differentiated cells have marked expression of neuron markers and hold the excitable properties of neurons, they can maintain their neuron-like morphology and neuronal marker expression stably. Therefore, these cells will be an important source of cells for transplantation studies for treating neurodegenerative diseases. 


\section{ACKNOWLEDGEMENTS}

This research was supported by the Guangdong Provincial Health Department Fund (No. A2011224), the National Natural Science Foundation of China (No. 30973693) and the National High Technology Research and Development Program (863 Program) (No. 2011AA02A111).

\section{REFERENCES}

1. J.A. Korecka, J. Verhaagen and E.M. Hol, Regen. Med., 2, 425 (2007)

2. V. Silani and M. Corbo, Curr. Neurovasc. Res., 1, 283 (2004).

3. C. du Pré Bastiaan, P.A. Doevendans and L.W. van Laake, J. Geriatr. Cardiol., 10, 186 (2013).

4. L.T. Lock and E.S. Tzanakakis, Tissue Eng., 13, 1399 (2007).

5. J.S. Yung, P.K. Tam and E.S. Ngan, Exp. Cell Res., 319, 177 (2013).

6. Z. Zhou, C.H. Chan, Q. Ma, X. Xu, Z. Xiao and E.K. Tan, Cell Adhes. Migr., 5, 280 (2011).

7. E.H. Javazon, K.J. Beggs and A.W. Flake, Exp. Hematol., 32, 414 (2004).

8. W.C. Low, W.W.Y. Yau, L.W. Stanton, G. Marcy, E. Goh and S.Y. Chew, DNA Cell Biol., 31, 1148 (2012).

9. T. Kondo, A.J. Matsuoka, A. Shimomura, K.R. Koehler, R.J. Chan, J.M. Miller, E.F. Srour and E. Hashino, Stem Cells, 29, 836 (2011).

10. P. Walczak, N. Chen, D. Eve, J. Hudson, T. Zigova, J. Sanchez-Ramos, P.R. Sanberg, C.D. Sanberg and A.E. Willing, Brain Res. Bull., 74, 155 (2007)

11. X. Li, P. Gong and D. Liao, Arch. Med. Sci., 6, 678 (2010).

12. S. Darabi, T. Tiraihi, A. Delshad and M. Sadeghizadeh, Iran. Biomed. J., 17, 8 (2013).

13. M. Munier, F. Law, G. Meduri, D. Le Menuet and M. Lombès, Endocrinology, 153, 1330 (2012).

14. F.M. Sanchez-Simon, X.X. Zhang, H.H. Loh, P.-Y. Law and R.E. Rodriguez, Mol. Pharmacol., 78, 935 (2010).

15. F.M.J. Jacobs, A.J.A. van der Linden, Y. Wang, L. von Oerthel, H.S. Sul, J.P.H. Burbach and M.P. Smidt, Development, 136, 2363 (2009).

16. F.M.J. Jacobs, S. van Erp, A.J.A. van der Linden, L. von Oerthel, J.P.H Burbach and M.P. Smidt, Development, 136, 531 (2009).

17. L.C. Lo, J.E. Johnson, C.W. Wuenschell, T. Saito and D.J. Anderson, Genes Dev., 5, 1524 (1991).

18. L. Ye, Z. Fan, B. Yu, J. Chang, K. Al Hezaimi, X. Zhou, N.-H. Park and C.-Y. Wang, Cell Stem Cell, 11, 50 (2012).
19. H. Sheng, X.- Rui, C.-J. Sheng, W.-J. Li, X.-Y. Cheng, N.P. Jhummon, Y.-C. Yu, S. Qu, G. Zhang and L. Qin, Int. J. Med. Sci., 10, 782 (2013).

20. A.M. Nelson, G.A. Larson and N.R. Zahniser, J. Pharmacol. Exp. Ther., 331, 985 (2009).

21. G.K. Vemuganti, N. Polisetti, V.G. Chaitanya and P.P. Babu, Neurol. India, 58, 201 (2010).

22. M. Kobune, Y. Kawano, Y. Ito, H. Chiba, K. Nakamura, H. Tsuda, K. Sasaki, H. Dehari, H. Uchida, O. Honmou, S. Takahashi, A. Bizen, R. Takimoto, T. Matsunaga, J. Kato, K. Kato, K. Houkin, Y. Niitsu and H. Hamada, Exp. Hematol., 31, 715 (2003).

23. C.-H. Park, J. Cell Sci., 119, 2310 (2006).

24. B. Joseph, A. Wallen-Mackenzie, G. Benoit, T. Murata, E. Joodmardi, S. Okret and T. Perlmann, Proc. Natl. Acad. Sci. USA, 100, 15619 (2003).

25. E.T. Courtois, C.G. Castillo, E.G. Seiz, M. Ramos, C. Bueno, I. Liste and A. Martínez-Serrano, J. Biol. Chem., 285, 9881 (2010).

26. E.T. Courtois, C.G. Castillo, E.G. Seiz, M. Ramos, C. Bueno, I. Liste and A. Martinez-Serrano, J. Biol. Chem., 285, 9881 (2010).

27. M. Florio, K. Leto, L. Muzio, A. Tinterri, A. Badaloni, L. Croci, P. Zordan, V. Barili, I. Albieri, F. Guillemot, F. Rossi and G.G. Consalez, Development, 139, 2308 (2012).

28. C. Hindley, F. Ali, G. McDowell, K. Cheng, A. Jones, F. Guillemot and A. Philpott, Development, 139, 1718 (2012).

29. A.W. Helms, Development, 132, 2709 (2005)

30. J. Li, J.A. Dani and W. Le, Curr. Top. Med. Chem., 9, 855 (2009).

31. G.-H. Guibinga, G. Hrustanovic, K. Bouic, H.A. Jinnah and T. Friedmann, Hum. Mol. Genet., 21, 609 (2012).

32. J.F. Reyes, Y. Fu, L. Vana, N.M. Kanaan and L.I. Binder, Am. J. Pathol., 178, 2275 (2011)

33. J.-M. Sontag, V. Nunbhakdi-Craig, C.L. White, S. Halpain and E. Sontag, J. Biol. Chem., 287, 14984 (2012).

34. M.E. Orr, R. Pitstick, B. Canine, K.H. Ashe and G.A. Carlson, PLoS ONE, 7, e39328 (2012).

35. I. Sanchez, L. Hassinger, R.K. Sihag, D.W. Cleveland, P. Mohan and R.A. Nixon, J. Cell Biol., 151, 1013 (2000).

36. M.H. Mokalled, A. Johnson, Y. Kim, J. Oh and E.N. Olson, Development, 137, 2365 (2010)

37. A. Yuan, M.V. Rao, Veeranna and R.A. Nixon, J. Cell Sci., 125, 3257 (2012).

38. H. Kanno, A. Kubo, T. Yoshizumi, T. Mikami and J. Maegawa, Int. J. Mol. Sci., 14, 9604 (2013).

39. D. Petrik, Y. Jiang, S.G. Birnbaum, C.M. Powell, M.-S. Kim, J. Hsieh and A.J. Eisch, FASEB J., 26, 3148 (2012). 